\title{
Teorias de Aprendizagem em Jogos Digitais Educacionais: um Panorama Brasileiro
}

\section{Theories of Learning in Educational Games: a Brazilian Overview}

\author{
Rafael João Ribeiro, IFPR, PPGECT-UTFPR - rafael.ribeiro@ifpr.edu.br \\ Nelson Silva Junior, UEPG, PPGECT-UTFPR - nelsonsj194@yahoo.com.br \\ Antonio Carlos Frasson, UTFPR - acfrasson@utfpr.edu.br \\ Luiz Alberto Pilatti, UTFPR - lapilatti@utfpr.edu.br \\ Sani de Carvalho Rutz da Silva, UTFPR - sani@utfpr.edu.br
}

\begin{abstract}
Resumo: Este estudo tem por objetivo identificar as teorias de aprendizagem aplicadas em jogos digitais educacionais desenvolvidos no Brasil no período de 2004 a 2014. Foram analisados 27 artigos selecionados com o auxílio da base de dados do Google Acadêmico, para isso, foram utilizados dois grupos de descritores. $\mathrm{Na}$ amostra, em $41 \%$ dos casos não foi identificada a aplicação de teorias de aprendizagem no desenvolvimento de jogos digitais educacionais. No restante da amostra, a teoria de Piaget é a mais citada, presente em 22\%. Em segundo, as teorias de Vygotsky aparecem em $15 \%$ das fundamentações teóricas. A concepção pedagógica adotada nas pesquisas da amostra condiz com o estado de arte internacional, isto é, uma preferência por teorias de base Construtivista. Entretanto, a Teoria de Aprendizagem Experiencial de Kolb, mais presente nas pesquisas internacionais, não é identificada na amostra.
\end{abstract}

Palavras-chave: jogos digitais educacionais, teorias de aprendizagem, instrumentos de avaliação.

Abstract: This study aims to identify the theories of learning applied to educational games research in Brazil in the period from 2004 to 2014. This study analyzed 27 articles found in the Google Scholar database using two groups of descriptors. It was verified that $41 \%$ of papers did not cite a theory of learning in the development of educational games. It was further observed that Piaget's theory, which was found in $22 \%$ of the articles, was the most frequently used theory. Vygotsky's theory appeared in $15 \%$ of the sample. The pedagogical principles adopted in Brazilian research corresponded to international "state of the art" because they reflected the Constructivist view of learning. However, Kolb's Experiential Learning Theory, more widely present in international research, was not mentioned in the sample of Brazilian research.

Keywords: educational digital games, learning theories, validated instruments.

\section{Introdução}

A aplicação de jogos digitais em propostas de ensino-aprendizagem é conhecida, em língua inglesa, como digital game-based learning (DGBL) (PRENSKY, 2007). Em língua portuguesa ainda não há um consenso sobre a terminologia, sendo uma alternativa: aprendizagem baseada em jogos digitais.

Hwang e Wu (2012) pesquisaram o estado da arte da tendência DGBL, numa perspectiva mundial, realizando uma revisão de 137 artigos publicados em sete periódicos da área entre 2001 e 2010. A pesquisa revelou que a tendência de publicações sobre DGBL é crescente, sendo que os três países com maior número de pesquisas no tema são: Estados Unidos, Inglaterra e China.

Para Bazzo et al. (2014), o desafio das escolas e dos educadores contemporâneos está em encontrar ferramentas que rompam com algumas tradições vigentes no ensino, promovendo mudanças estruturais na chamada educação tecnológica, da qual os jogos digitais fazem parte.

Os jogos usados em sala de aula podem se apresentar de duas formas: aqueles que foram criados com fins educacionais e se destinam a um uso específico para determinada disciplina ou conteúdo ou aqueles comerciais que o professor consegue estabelecer uma relação entre o conteúdo a ser trabalhado e o conteúdo do jogo (LACASA; MÉNDEZ e MARTÍNEZ, 2008).

Tanto o uso de um, quanto do outro, exige do professor um preparo especial para que se possa transpor, didaticamente, conteúdos e informações da disciplina e do jogo, não perdendo de vista que o jogo tem características próprias de entretenimento e que estas, apesar de fundamentais, não devem se sobrepor ao caráter educacional do jogo, quando utilizado com este fim (OKAN, 2003). 
Para auxiliar professores na escolha de jogos digitais educacionais, Freitas e Oliver (2006) elaboraram um guia para o reconhecimento dos aspectos pedagógicos presentes em jogos digitais, enfatizando a necessidade de identificação de uma concepção pedagógica que atenda os objetivos e resultados esperados no processo de aprendizagem.

Um estudo da arte das concepções pedagógicas utilizadas em jogos digitais, com propósitos pedagógicos, foi realizado por $\mathrm{Wu}$ et al. (2012). No levantamento foram identificadas as teorias de aprendizagem adotadas como concepção pedagógica na construção de jogos digitais educacionais. Usando unitermos específicos com a palvra "game" e as palavras chave pedagogy, instruct, learn, teach, training, entertainment, play, computer game, learning strategies, e design, os autores encontraram 567 estudos sobre aprendizagem baseada em jogos digitais em importantes bases de dados como ACM Digital Library, ERIC, SAGE, ScienceDirect, SpringerLink, Taylor and Francis Online e Wiley, dos quais, considerando os critérios de inclusão e exclusão estabelecidos, 91 foram selecionados. O Quadro 1 sumariza os resultados encontrados por Wu et al. (2012).

\begin{tabular}{|c|c|c|c|}
\hline $\begin{array}{l}\text { Concepçãa } \\
\text { pedagógica }\end{array}$ & Teoria de aprendizagem & Representante & $\begin{array}{c}\mathbf{N}^{\circ} \text { de } \\
\text { publicações }\end{array}$ \\
\hline \multirow{3}{*}{$\begin{array}{l}\text { Behaviorismo } \\
(13 \%)\end{array}$} & Instrução direta & Zig Engelmann (1960) & 9 \\
\hline & Instrução programada & Skinner (1954) & 3 \\
\hline & Teoria da aprendizagem social & Bandura (1977) & 3 \\
\hline \multirow{4}{*}{$\begin{array}{l}\text { Cognitivismo } \\
(14 \%)\end{array}$} & Teoria da atribuição causal & Weiner (1974) & 7 \\
\hline & Teoria da elaboração & Reigeluth (1983) & 4 \\
\hline & Desenvolvimento cognitivo & Piaget (1969) & 3 \\
\hline & Condições de aprendizagem & Gagné (1965) & 2 \\
\hline Humanismo (22\%) & $\begin{array}{l}\text { Teoria da aprendizagem } \\
\text { experiencial }\end{array}$ & Kolb (1984) & 25 \\
\hline \multirow[t]{8}{*}{$\begin{array}{l}\text { Construtivismo } \\
(51 \%)\end{array}$} & $\begin{array}{l}\text { Teoria do desenvolvimento } \\
\text { social }\end{array}$ & Vygostsky (1962) & 17 \\
\hline & $\begin{array}{l}\text { Aprendizagem baseada em } \\
\text { problemas }\end{array}$ & $\begin{array}{l}\text { Originou em escolas de medicina na } \\
\text { década de } 1960 .\end{array}$ & 13 \\
\hline & Aprendizagem cognitiva & Vygotsky (1978) & 8 \\
\hline & Aprendizagem por descoberta & Bruner $(1960)$ & 7 \\
\hline & Aprendizagem baseada em casos & $\begin{array}{l}\text { Originou da aprendizagem baseada em } \\
\text { problemas na década de } 1990 .\end{array}$ & 5 \\
\hline & Aprendizagem situada & Lave e Wenger (1990) & 4 \\
\hline & $\begin{array}{l}\text { Aprendizagem como atividade } \\
\text { lúdica }\end{array}$ & Leont'ev (1978) & 3 \\
\hline & $\begin{array}{l}\text { Aprendizagem sob a perspectiva } \\
\text { da teoria ator-rede }\end{array}$ & Latour (1987) & 1 \\
\hline
\end{tabular}

Quadro 1 - Classificação de concepções e teorias de aprendizagem presentes em artigos sobre DGBL.

Fonte: Adaptado de Wu et al. (2012, p.1158)

O presente estudo, usando unitermos em português e o Google acadêmico para a constituição do corpus documental, percorreu caminho similar ao de Wu et al. (2012). Com esse contorno, o presente estudo tem por objetivo identificar as teorias de aprendizagem aplicadas em jogos digitais educacionais desenvolvidos no Brasil, voltados para o ensino básico, no período de 2004 a 2014.

\section{Metodologia}

O presente estudo, de natureza descritiva, configura-se como uma revisão sistemática da literatura, sem metanálise, utilizando-se os mecanismos de busca do Google Acadêmico para a constituição do corpus documental. Inicialmente, foi realizada uma busca utilizando os seguintes unitermos: "jogo digital", "jogo de computador", "jogo eletrônico" e "jogo virtual". Adotou-se como critério de inclusão considerar apenas os artigos que continham as expressões no título do artigo.

Na sequência, uma segunda busca foi realizada com os unitermos "jogo educativo" e "jogo educacional", presentes no título dos trabalhos. Como primeiro critério de seleção, apenas os artigos que apresentavam o nome do jogo no título do artigo foram considerados. 
Considerando a abrangência desses unitermos, uma análise do conteúdo de cada artigo foi realizada para verificar se o jogo tratado era no formato digital e se atendia objetivo do estudo.

Em ambas as buscas, como critério de inclusão, foram selecionados artigos completos de acesso livre, publicados no idioma português, em eventos ou periódicos, resultantes de pesquisas realizadas no Brasil no período de 2004 a 2014. Os artigos da amostra foram lidos e analisados, principalmente, quanto à presença ou ausência de teorias de aprendizagem norteadoras na decisão do design do estudo.

O procedimento de análise dos artigos envolveu a identificação e busca das seguintes informações: título do jogo; plataforma de distribuição; ferramenta de desenvolvimento do jogo; conteúdo pedagógico abordado; público alvo; concepção pedagógica ou teoria de aprendizagem norteadora; e instrumento de avaliação.

\section{Resultados}

Na primeira busca, após a leitura de cada trabalho, constatou-se que 105 resultados não atendiam aos objetivos da pesquisa. Assim, identificou-se 26 pesquisas que tratam de jogos digitais não comerciais e independentes. No final, a amostra resultante da primeira busca foi formada por 15 artigos completos que tratam de jogos digitais educacionais produzidos para atender objetivos pedagógicos específicos (Quadro 2).

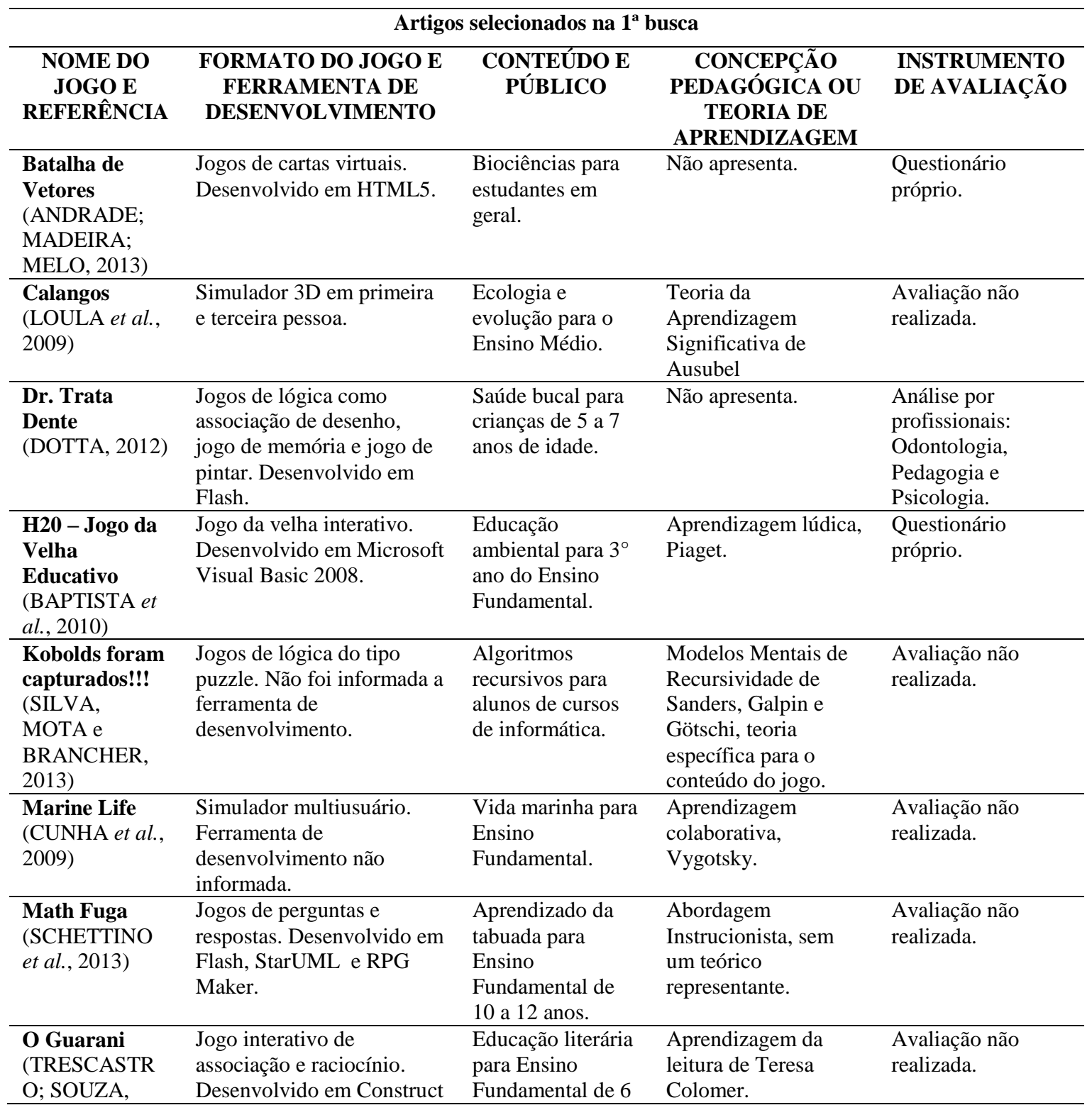




\begin{tabular}{|c|c|c|c|c|}
\hline 2014) & 2. & a 8 anos. & & \\
\hline $\begin{array}{l}\text { O Olho: } \\
\text { surreality } \\
\text { (SALOMÃO; } \\
\text { HEIDRICH, } \\
\text { 2007) }\end{array}$ & $\begin{array}{l}\text { Jogo de aventura interativo. } \\
\text { Desenvolvido em Flash. }\end{array}$ & $\begin{array}{l}\text { Movimento } \\
\text { Surrealista para } \\
\text { estudantes em } \\
\text { geral. }\end{array}$ & Não apresenta. & $\begin{array}{l}\text { Avaliação não } \\
\text { realizada. }\end{array}$ \\
\hline $\begin{array}{l}\text { Pedrinho em: } \\
\text { conhecendo o } \\
\text { mapa } \\
\text { (DAMBROS et } \\
\text { al., 2013) }\end{array}$ & $\begin{array}{l}\text { Multimídia interativo } \\
\text { desenvolvido em Flash. }\end{array}$ & $\begin{array}{l}\text { Cartografia para } 5^{\mathrm{a}} \\
\text { série do Ensino } \\
\text { Fundamental. }\end{array}$ & Não apresenta. & $\begin{array}{l}\text { Questionário } \\
\text { próprio. }\end{array}$ \\
\hline $\begin{array}{l}\text { Pense bem } \\
\text { (MELO; } \\
\text { COSTA; } \\
\text { BATISTA, } \\
\text { 2013) }\end{array}$ & $\begin{array}{l}\text { Jogo de tabuleiro virtual. } \\
\text { Desenvolvido em Construct } \\
2 \text {. }\end{array}$ & $\begin{array}{l}\text { Computação para } \\
\text { Ensino Médio e } \\
\text { Fundamental a } \\
\text { partir de } 12 \text { anos. }\end{array}$ & $\begin{array}{l}\text { Taxonomia revisada } \\
\text { de Bloom. }\end{array}$ & $\begin{array}{l}\text { Avaliação não } \\
\text { realizada. }\end{array}$ \\
\hline $\begin{array}{l}\text { QUIZmica } \\
\text { (LUCENA; } \\
\text { AZEVEDO, } \\
\text { 2012) }\end{array}$ & $\begin{array}{l}\text { Jogo de perguntas e } \\
\text { respostas desenvolvido no } \\
\text { Microsoft Power Point } \\
2007 .\end{array}$ & $\begin{array}{l}\text { Química geral } \\
\text { experimental para } \\
1^{\mathrm{a}} \text { série do Ensino } \\
\text { Médio. }\end{array}$ & $\begin{array}{l}\text { Aprendizagem } \\
\text { Lúdica. Piaget e } \\
\text { Vygotsky. }\end{array}$ & $\begin{array}{l}\text { Questionário } \\
\text { próprio. }\end{array}$ \\
\hline $\begin{array}{l}\text { Sherlock } \\
\text { Dengue } \\
\text { (BUCHINGER; } \\
\text { HOUNSELL; } \\
\text { DIAS, 2012) } \\
\end{array}$ & $\begin{array}{l}\text { Jogo 3D em primeira } \\
\text { pessoa. Desenvolvido em } \\
\text { JAVA e Xj3D. }\end{array}$ & $\begin{array}{l}\text { Doenças } \\
\text { infecciosas para } \\
\text { estudantes em } \\
\text { geral. }\end{array}$ & Não apresenta. & $\begin{array}{l}\text { Adaptado de outra } \\
\text { fonte. }\end{array}$ \\
\hline $\begin{array}{l}\text { Tríade } \\
\text { (SANTANA et } \\
\text { al., 2007) }\end{array}$ & $\begin{array}{l}\text { Jogo de aventura. } \\
\text { Ferramenta de } \\
\text { desenvolvimento não } \\
\text { informada. }\end{array}$ & $\begin{array}{l}\text { Revolução } \\
\text { Francesa para } 8^{\text {a }} \\
\text { série do Ensino } \\
\text { Fundamental. } \\
\end{array}$ & $\begin{array}{l}\text { Aprendizagem lúdica } \\
\text { e teorias } \\
\text { psicogenéticas. } \\
\text { Vygotsky e Piaget. }\end{array}$ & $\begin{array}{l}\text { Avaliação não } \\
\text { realizada. }\end{array}$ \\
\hline $\begin{array}{l}\text { UruBurbanos } \\
\text { (AZEVEDO } \text { et } \\
\text { al., 2011) }\end{array}$ & $\begin{array}{l}\text { Jogo 3D em terceira pessoa. } \\
\text { Ferramenta de } \\
\text { desenvolvimento não } \\
\text { informada. }\end{array}$ & $\begin{array}{l}\text { Educação } \\
\text { ambiental para } \\
\text { Ensino } \\
\text { Fundamento e } \\
\text { Médio. }\end{array}$ & Não apresenta. & $\begin{array}{l}\text { Questionário } \\
\text { próprio. }\end{array}$ \\
\hline
\end{tabular}

Quadro 2 - Análise da amostra de 15 artigos sobre desenvolvimento e aplicação de jogos digitais educacionais em pesquisas brasileiras.

Na segunda busca, como critério de inclusão, apenas as pesquisas que apresentavam no título o nome do jogo em análise foram consideradas. Assim, dos 191 resultados, após aplicação do critério de seleção, após a análise de cada item, foram encontrados 12 pesquisas que atendem os objetivos desse estudo. Essa seleção envolveu a leitura dos artigos, pois os descritores utilizados também representam outros tipos de jogos, tais como jogos de tabuleiro, jogos de carta, entre outros (Quadro 3).

\begin{tabular}{|c|c|c|c|c|}
\hline \multicolumn{5}{|c|}{ Artigos selecionados na $2^{\mathrm{a}}$ busca } \\
\hline $\begin{array}{l}\text { Nome do Jogo } \\
\text { e Referência }\end{array}$ & $\begin{array}{l}\text { Formato do jogo e } \\
\text { ferramenta de } \\
\text { desenvolvimento }\end{array}$ & Conteúdo e público & $\begin{array}{l}\text { Concepção pedagógica } \\
\text { ou Teoria de } \\
\text { Aprendizagem }\end{array}$ & $\begin{array}{l}\text { Instrumento } \\
\text { de validação }\end{array}$ \\
\hline $\begin{array}{l}\text { Digita } \\
\text { (AN et al., } \\
2013) \\
\end{array}$ & $\begin{array}{l}\text { Jogo interativo. } \\
\text { Desenvolvido em C\#, na } \\
\text { plataforma Dot Net. }\end{array}$ & $\begin{array}{l}\text { Alfabetização para } \\
\text { crianças. }\end{array}$ & $\begin{array}{l}\text { Método Paulo Freire de } \\
\text { alfabetização. }\end{array}$ & $\begin{array}{l}\text { Avaliado por } \\
\text { especialistas. }\end{array}$ \\
\hline $\begin{array}{l}\text { EducaTrans } \\
\text { (ASSIS } \text { et al., } \\
\text { 2006) }\end{array}$ & $\begin{array}{l}\text { Jogo 3D multiusuário. } \\
\text { Desenvolvido em } \\
\text { Blender 3D }\end{array}$ & $\begin{array}{l}\text { Educação no } \\
\text { Trânsito para o } \\
\text { Ensino Fundamental } \\
\text { e Ensino Médio }\end{array}$ & $\begin{array}{l}\text { Taxonomia dos objetivos } \\
\text { educacionais de Bloom. }\end{array}$ & $\begin{array}{l}\text { Avaliação não } \\
\text { realizada. }\end{array}$ \\
\hline $\begin{array}{l}\text { Elimine a } \\
\text { Dengue } \\
\text { (SOUSA; } \\
\text { GUIMARÃES; } \\
\text { PIRES, 2012) }\end{array}$ & $\begin{array}{l}\text { Jogo interativo. } \\
\text { Desenvolvido em Flash. }\end{array}$ & $\begin{array}{l}\text { Dengue para público } \\
\text { infantil. }\end{array}$ & Não apresenta. & $\begin{array}{l}\text { Metodologia } \\
\text { observacional e } \\
\text { entrevistas. }\end{array}$ \\
\hline $\begin{array}{l}\text { Exterminadore } \\
\text { s de Dengue } \\
\text { (SILVA, Valter }\end{array}$ & $\begin{array}{l}\text { Jogo 2D de aventura. } \\
\text { Desenvolvido em RPG } \\
\text { Maker VX e XNA. }\end{array}$ & $\begin{array}{l}\text { Combate à Dengue } \\
\text { para Ensino } \\
\text { Fundamental. }\end{array}$ & Não apresenta. & $\begin{array}{l}\text { Avaliação não } \\
\text { realizada. }\end{array}$ \\
\hline
\end{tabular}




\begin{tabular}{|c|c|c|c|c|}
\hline da et al., 2011) & & & & \\
\hline $\begin{array}{l}\text { Geonandia 3D } \\
\text { (BRUM; } \\
\text { SILVEIRA, } \\
\text { 2004) }\end{array}$ & $\begin{array}{l}\text { Jogo em 3D e 2.5D. } \\
\text { Ferramenta de } \\
\text { desenvolvimento não } \\
\text { informada. }\end{array}$ & $\begin{array}{l}\text { Geometria para } 4^{\mathrm{a}} \\
\text { série do Ensino } \\
\text { Fundamental. }\end{array}$ & Não apresenta. & $\begin{array}{l}\text { Questionários } \\
\text { próprios. }\end{array}$ \\
\hline $\begin{array}{l}\text { IntEducaTrâns } \\
\text { ito } \\
\text { (MACEDO et } \\
\text { al., 2013) }\end{array}$ & $\begin{array}{l}\text { Jogo 3D simulador de } \\
\text { auto-escola. } \\
\text { Desenvolvido em } \\
\text { Blender 3D. }\end{array}$ & $\begin{array}{l}\text { Normas de trânsito } \\
\text { para Ensino } \\
\text { Fundamental e } \\
\text { Médio. }\end{array}$ & Não apresenta. & $\begin{array}{l}\text { Avaliação não } \\
\text { realizada. }\end{array}$ \\
\hline $\begin{array}{l}\text { Labirinto do } \\
\text { Rato } \\
\text { (SILVA, } \\
\text { Sebastian } \\
\text { Saullo Ribeiro } \\
\text { da; NÓBREGA; } \\
\text { Jacob Jr, 2011) }\end{array}$ & $\begin{array}{l}\text { Jogo de labirinto. } \\
\text { Desenvolvido em sistema } \\
\text { Android. }\end{array}$ & $\begin{array}{l}\text { Raciocínio lógico } \\
\text { para público infantil. }\end{array}$ & $\begin{array}{l}\text { Aprendizagem lúdica, } \\
\text { Vygotsky. }\end{array}$ & $\begin{array}{l}\text { Avaliação não } \\
\text { realizada. }\end{array}$ \\
\hline $\begin{array}{l}\text { Laguna } \\
\text { (MÜLLER; } \\
\text { CAVALCANT } \\
\text { E; SANTOS, } \\
\text { 2007) } \\
\end{array}$ & $\begin{array}{l}\text { Jogo 3D de aventura. } \\
\text { Desenvolvido no } \\
\text { Blender. }\end{array}$ & $\begin{array}{l}\text { Guerra do Paraguai } \\
\text { para Ensino } \\
\text { Fundamental de } 13 \text { a } \\
15 \text { anos. }\end{array}$ & $\begin{array}{l}\text { Construtivismo de Piaget } \\
\text { e o construcionismo de } \\
\text { Papert. }\end{array}$ & $\begin{array}{l}\text { Avaliação não } \\
\text { realizada. }\end{array}$ \\
\hline $\begin{array}{l}\text { SoletrandoMo } \\
\text { b } \\
\text { (MESQUITA } \\
\text { FILHO, 2010) }\end{array}$ & $\begin{array}{l}\text { Jogo de soletração para } \\
\text { celular. Desenvolvido em } \\
\text { Java ME e LWUIT. }\end{array}$ & $\begin{array}{l}\text { Ensino ortográfico } \\
\text { da língua portuguesa } \\
\text { para estudantes a } \\
\text { partir de } 10 \text { anos e } \\
\text { adultos. }\end{array}$ & Construtivismo de Piaget. & $\begin{array}{l}\text { Avaliação não } \\
\text { realizada. }\end{array}$ \\
\hline $\begin{array}{l}\text { Spacequiz } \\
\text { (SILVA, } \\
\text { Francisco } \\
\text { Airton Pereira } \\
\text { da, 2009) } \\
\end{array}$ & $\begin{array}{l}\text { Jogo de ação para } \\
\text { celular. Desenvolvido em } \\
\text { J2ME. }\end{array}$ & $\begin{array}{l}\text { Aritmética para } \\
\text { Ensino } \\
\text { Fundamental. }\end{array}$ & Construtivismo de Piaget. & $\begin{array}{l}\text { Questionários } \\
\text { próprios. }\end{array}$ \\
\hline $\begin{array}{l}\text { Terra das } \\
\text { Cores } \\
\text { (LEITÃO et al., } \\
\text { 2012) } \\
\end{array}$ & $\begin{array}{l}\text { Jogo 2D de gêneros } \\
\text { puzzle e aventura. } \\
\text { Desenvolvido em } \\
\text { Blender e XNA. }\end{array}$ & $\begin{array}{l}\text { Matemática para } \\
\text { crianças. }\end{array}$ & $\begin{array}{l}\text { Teoria das Inteligências } \\
\text { Múltiplas de Gardner. }\end{array}$ & $\begin{array}{l}\text { Avaliação não } \\
\text { realizada. }\end{array}$ \\
\hline $\begin{array}{l}\text { URIHI } \\
\text { (CAMPOS et } \\
\text { al., 2012) }\end{array}$ & $\begin{array}{l}\text { Jogo 2D. Desenvolvido } \\
\text { em Game Maker 8.1. }\end{array}$ & $\begin{array}{l}\text { Preservação } \\
\text { ambiental para } \\
\text { Ensino } \\
\text { Fundamental. } \\
\end{array}$ & Não apresenta. & $\begin{array}{l}\text { Avaliação não } \\
\text { realizada. }\end{array}$ \\
\hline
\end{tabular}

Agrupando os artigos encontrados nas duas etapas, a amostra dessa revisão sistemática da literatura é formada por 27 pesquisas brasileiras que tratam do desenvolvimento e aplicação de jogos digitais educacionais (Quadro 23).

Constatou-se que 40,74\% ( $\mathrm{n}=11)$ dos jogos digitais educacionais apresentados em pesquisas brasileiras não apresentam explicitamente teorias de aprendizagem na fundamentação teórica- Nas demais, observa-se que a teoria de Piaget é a mais citada, presente em 22,22\%, $(n=6)$ seguida das teorias de Vygotsky, que aparecem em 14,81\% (n=4) das fundamentações teóricas. Além dessas, foram identicadas as seguintes teorias: Bloom $(7,40 \% ; n=2)$; Ausubel $(3,70 \% ; n=1)$; Papert $(3,70 \% ; n=1)$; Gardner $(3,70 \% ; n=1)$; Paulo Freire $(3,70 \% ; n=1)$; Teresa Colomer $(3,70 \% ; n=1)$; abodagem instrucionista, sem um teórico representante $(3,70 \% ; n=1)$. Também, constatou-se que 59,26\% (n=16) dos artigos não relatam a aplicação de instrumentos para a verificação dos objetivos pedagógicos dos jogos.

Um histograma da distribuição por ano de publicação dos artigos em intervalo de dois anos é apresentado no Gráfico 1. Conforme a amostra, observa-se um aumento significativo de pesquisas brasileiras sobre jogos digitais educacionais principalmente nos últimos dois anos. 
Distribuição da amostra por ano de publicação

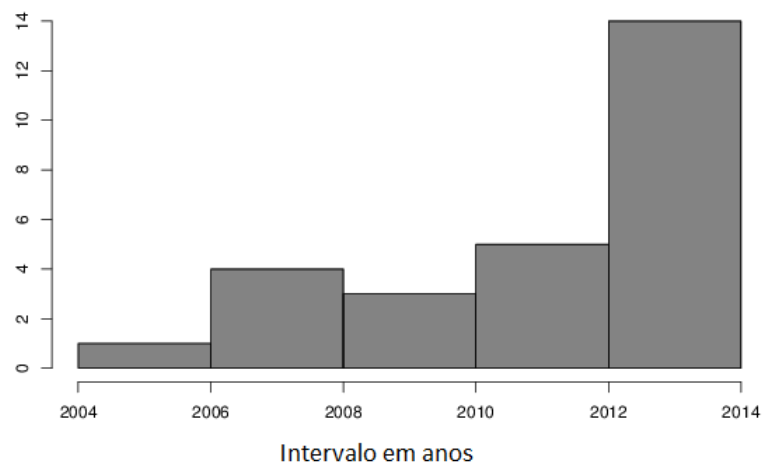

Gráfico 1 - Distribuição da amostra por ano de publicação. Fonte: autoria própria.

\section{Discussão}

A análise dos 27 artigos que compõem o corpus documental mostra que 40,74\% das pesquisas não se preocupam com a adoção de uma teoria de aprendizagem que fundamente $o$ desenvolvimento e a aplicação dos jogos. A ausência de uma teoria de aprendizagem na fundamentação de um jogo interfere, diretamente, no seu sucesso como mediador da aprendizagem (WIDEMAN et al., 2007). Nas demais pesquisas, observa-se que a teoria construtivista de Piaget é a mais utilizada, presente em 22,22\% das pesquisas brasileiras sobre jogos digitais educacionais. Em segundo, as teorias de Vygotsky aparecem em 14,81\% das fundamentações teóricas.

Assim, entre as teorias da aprendizagem identificadas, observa-se um destaque para as postuladas por Piaget e Vygotsky. Apesar deste estudo não ter vivenciado a prática dos jogos que foram objetos das publicações, pode-se observar que as pesquisas que revelam uma Teoria da Aprendizagem partem de um pressuposto didático pedagógico, fundamentado em questões sobre o desenvolvimento cognitivo do homem e que, consequentemente, podem representar um avanço no processo de ensino e aprendizagem de uma determinada área do conhecimento.

Jean Piaget, epistemólogo suíço, considerava as ações do homem como a base de todo comportamento humano. Para ele, ensinar supõe criar situações compatíveis com o nível de desenvolvimento da criança, que venham acompanhadas de ações e demonstrações integradas à prática pedagógica do professor. Quando pensamos em seus pressupostos relacionados ao uso de jogos digitais educacionais, estes nos remetem aos esquemas de assimilação que, segundo Piaget, a criança utiliza no seu desenvolvimento intelectual e o quanto os jogos trabalham na perspectiva de esquemas de assimilação para avançar nas etapas propostas pelo jogo.

Lev Vygotsky, cientista bielorrusso, desenvolveu suas teorias a partir do pressuposto que o desenvolvimento cognitivo do ser humano não pode ser entendido sem as referências ao contexto social e cultural no qual o homem está inserido. A aprendizagem acontece mediada por fatores sociais, históricos e culturais, que convivem no espaço onde as relações de ensino e aprendizagem acontecem. A cultura digital na qual estamos imersos é um desses fatores apontados por Vygotsky, tendo os jogos digitais educacionais papel fundamental nas ações interacionistas que aproximam o estudante da escola, aqui entendida num sentido mais amplo. A criação de um jogo educacional que parte de um referencial interacionista, como a teoria defendida por Vygotsky, tem na essência de sua concepção bases didático pedagógicas que vão consolida-lo como ferramenta consistente de ensino e não apenas como mais um elemento usado para diversificação no aprendizado.

Apesar da baixa diversidade, a concepção pedagógica adotada em pesquisas brasileiras sobre jogos digitais educacionais condiz com o estado de arte internacional (WU et al., 2012), que também apresenta uma preferência pela aplicação de teorias de aprendizagem de base Construtivista. Entretanto, a Teoria de Aprendizagem Experiencial de Kolb, a mais presente nas pesquisas internacionais, não é citada na amostra de pesquisas brasileiras.

$\mathrm{Na}$ amostra, uma diferença encontrada nas pesquisas brasileiras é que os jogos digitais educacionais não passam por processos de validação em 59,26\% dos casos. Além disso, nos 
demais trabalhos da amostra, as metodologias de avaliação de jogos digitais educacionais não aplicam instrumentos estatísticos validados, que apresentem confiabilidade. Para tanto, existem instrumentos, como questionários, que passam por processos de testagem de propriedades psicométricas (PILATTI; PEDROSO e GUTIERREZ, 2010). No caso da amostra desta pesquisa não há indicação de uso desse tipo de instrumento.

O emprego de escalas de avaliação de jogos digitais educacionais permite que professores possam identificar a qualidade de um jogo, como elemento relevante no processo de ensino e aprendizagem, antes da sua aplicação em sala de aula (AK, 2012). Como exemplo, quatro instrumentos validados que apresentam confiabilidade estatística e que podem ser reaplicados em pesquisas sobre DGBL: eGameFlow (FU; SU e YU, 2009); Game Engagement Questionnaire (GEQ) (BROCKMYER et al, 2009); Social Presence in Gaming Questionnaire (SPGQ) (KORT; IJSSELSTEIJN e POELS, 2007); Instructional Material Motivational Survey (IMMS) (HUANG et al., 2006).

A falta de adoção de teorias de aprendizagem em 40,74\% da amostra e a falta de aplicação de instrumentos validados e confiáveis na avaliação em pesquisas brasileiras é uma situação que não colabora para a consolidação do emprego da aprendizagem baseada em jogos digitais em ambientes de ensino. Pois, jogos desenvolvidos sem uma concepção pedagógica de referência podem produzir resultados distintos dos concebidos.

Considerando o resultado do presente estudo, o ceticismo à inserção dos jogos em ambientes de ensino, em alguma medida, justifica-se. Conforme resumido por Prensky (2012), os céticos ou negadores podem ser divididos em seis grupos: (1) os tradicionalistas; (2) os revisores e reforçadores; e (3) o grupo do não vale a pena; (4) o grupo do eu receio; (5) o grupo dos idealistas; e (6) o grupo dos pessimistas.

Os jogos digitais são comumente associados a uma atividade de entretenimento e criticados por seus elementos atrativos capazes de manter um jogador por horas diante da tela do jogo (PORTER et al., 2010). Na maioria dos casos, os jogadores buscam algum tipo de retorno pessoal, como prazer em vencer desafios e a obtenção de bons resultados, ou ainda, retorno social, no caso dos jogos online (YEE, 2006).

Essa característica de atração intrínseca aos jogos digitais também facilita a sua aplicação com finalidades educacionais, seja de aprendizagem, conscientização ou socialização, como ocorre no caso dos jogos do gênero serious games, que são jogos de propósitos sérios com objetivos de conscientização para um problema social real (BREUER e BENTE, 2010).

\section{Considerações finais}

Os Jogos Digitais Educacionais ainda se apresentam como uma novidade no espaço escolar, seja pela inexistência de jogos que atendam especificamente aos processos de ensino e aprendizagem de uma determinada área do conhecimento, seja pelo desconhecimento das possibilidades de uso neste campo.

A inserção de ferramentas científico tecnológicas nas relações de aprendizagem, ainda que de forma lenta, trazem uma nova revolução no ensino contemporâneo. Vale lembrar que quando o giz em bastão chegou às salas de aula no século XIX, ele representava um avanço tecnológico importante para o campo da educação e seu uso foi sendo incorporado ao cotidiano escolar de tal forma que sua ausência chegou a ser inadmissível para qualquer professor e um objeto de desejo, disputado por alunos. Hoje, para muitos professores, o giz está superado e foi substituído por inúmeros outros aparatos e alguns alunos quase que o desconhecem.

Os Jogos Digitais Educacionais vêm ganhando notoriedade na escola. O crescimento das publicações, no meio acadêmico, sobre o tema no Brasil em 10 anos (2004 - 2014) alinhase, provavelmente, com a notoriedade mencionada. Observou-se que, apesar do crescente uso do jogo como parte integrante do processo ensino aprendizagem, para diferentes áreas do conhecimento, a elaboração, o desenvolvimento e a aplicação desses jogos acontecem, ainda, de forma desvinculada às Teorias de Aprendizagem, em boa parte dos casos aqui estudados.

Considerando que uma Teoria da Aprendizagem pode apontar como abordar um assunto, a sua referência teórica na construção de um jogo educativo, em especial o digital, torna o jogo uma ferramenta mais consistente na prática pedagógica. O professor, que ao mediar 
o seu uso nas relações escolares de ensino e aprendizagem, o fará a partir de uma perspectiva educacional e não meramente ilustrativa ou de entretenimento.

A amostra analisada neste estudo revelou que os jogos digitais educacionais divulgados em pesquisas brasileiras não contemplam, em 40,74\% dos casos, uma preocupação com o emprego de teorias de aprendizagem. A análise também mostra uma baixa diversidade de teorias de aprendizagem aplicadas em jogos brasileiros, sendo a teoria de Piaget e a de Vygotsky as mais utilizadas. Considerando a amostra brasileira analisada, a escolha de concepções pedagógicas para a fundamentação de jogos digitais condiz com o estado de arte internacional, ou seja, há uma preferência por teorias de aprendizagem de base Construtivista.

\section{Referências}

AK, Oguz. A game scale to evaluate educational computer games. Social and Behavioral Sciences, v. 46, p. 2477-2481, jan. 2012.

BAZZO, Walter Antonio; BAZZO, Jilvania Lima dos Santos; PEREIRA, Luiz Teixeira do Vale. Conversando sobre educação tecnológica. Florianópolis: Editora da UFSC, 2014.

BREUER, Johannes; BENTE, Gary. Why so serious? On the relation of serious games and learning. Eludamos. Journal for Computer Game Culture, v. 4, n. 1, p. 7-24, 2010.

BROCKMYER, Jeanne H. et al. The development of the Game Engagement Questionnaire: A measure of engagement in video game-playing. Journal of Experimental Social Psychology, v. 45, n. 4, p. 624-634, jul. 2009.

FREITAS, Sara de; OLIVER, Martin. How can exploratory learning with games and simulations within the curriculum be most effectively evaluated? Computers \& Education, v. 46, n. 3, p. 249-264, abr. 2006.

FU, Fong-Ling; SU, Rong-Chang; YU, Sheng-Chin. EGameFlow: A scale to measure learners' enjoyment of e-learning games. Computers \& Education, v. 52, n. 1, p. 101-112, jan. 2009.

HUANG, Wenhao et al. A preliminary validation of Attention, Relevance, Confidence and Satisfaction model-based Instructional Material Motivational Survey in a computer-based tutorial setting. British Journal of Educational Technology, v. 37, n. 2, p. 243-259, mar. 2006.

HWANG, Gwo-Jen; WU, Po-Han. Advancements and trends in digital game-based learning research: a review of publications in selected journals from 2001 to 2010. British Journal of Educational Technology, v. 43, n. 1, p. E6-E10, 21 jan. 2012.

KOLB, David. A. Experiential learning: experience as the source of learning and development. Englewood Cliffs, NJ: Prentice Hall, 1984.

KORT, Y.A.W. de; IJSSELSTEIJN, W. A.; POELS, K. Digital Games as Social Presence Technology: Development of the Social Presence in Gaming Questionnaire (SPGQ). In: PRESENCE, 2007, Barcelona. Anais. Barcelona: Starlab e PEACH., 2007. p. 195-203.

LACASA, Pilar; MÉNDEZ, Laura; MARTÍNEZ, Rut. Bringing Commercial Games into the Classroom. Computers and Composition, v. 25, n. 3, p. 341-358, jan. 2008.

OKAN, Zuhal. Edutainment: is learning at risk? British Journal of Educational Technology, v. 34, n. 3, p. 255-264, jun. 2003.

PILATTI, Luiz Alberto; PEDROSO, Bruno; GUTIERREZ, Gustavo Luis. Propriedades psicométricas de instrumentos de avaliação: um debate necessário. Revista Brasileira de Ensino de Ciência e Tecnologia, v. 3, n. 1, 2010.

PORTER, Guy et al. Recognizing problem video game use. The Australian and New Zealand journal of psychiatry, v. 44, n. 2, p. 120-8, fev. 2010.

PRENSKY, Marc. Aprendizagem baseada em jogos digitais. São Paulo: SENAC, 2012. p. 575

PRENSKY, Marc. The Digital Game-Based Learning Revolution. Minnesota: Paragon House, 2007. 
WIDEMAN, H. H. et al. Unpacking the potential of educational gaming: A new tool for gaming research. Simulation \& Gaming, v. 38, n. 1, p. 10-30, 1 mar. 2007.

WU, Wen-Hsiung et al. Re-exploring game-assisted learning research: The perspective of learning theoretical bases. Computers \& Education, v. 59, n. 4, p. 1153-1161, dez. 2012.

YEE, Nick. Motivations for play in online games. Cyberpsychology \& behavior : the impact of the Internet, multimedia and virtual reality on behavior and society, v. 9, n. 6, p. 772-5, dez. 2006.

\section{Anexo \\ Corpus documental}

AN, Ding Yih et al. Digita - um Jogo Educativo de Apoio ao Processo de Alfabetização Infantil. In: CONGRESSO BRASILEIRO DE INFORMÁTICA NA EDUCAÇÃO (CBIE 2013) XXIV SIMPÓSIO BRASILEIRO DE INFORMÁTICA NA EDUCAÇÃO (SBIE 2013). 2., 2013, Campinas. Anais. Campinas: Unicamp, 22 nov. 2013. p. 154-163.

ANDRADE, Adja F. de; MADEIRA, Chareles Andryê G.; MELO, Higor Hícaro A. R. F. Batalha de Vetores Virtual: uma proposta de jogo pedagógico para o ensino de biociências. Nuevas Ideas en Informática Educativa TISE, p. 105-112, 2013.

ASSIS, Gilda Aparecida de et al. EducaTrans: um Jogo Educativo para o Aprendizado do Trânsito. Novas Tecnologias na Educação, v. 4, n. 2, p. 1-10, 2006.

AZEVEDO, Ryan Ribeiro de et al. Ensino e Aprendizagem de Educação Ambiental Através de um Jogo Eletrônico. In: XXII SIMPÓSIO BRASILEIRO DE INFORMÁTICA NA EDUCAÇÃO - XVII WIE, 12., 2011, Aracajú. Anais. Aracajú: Sociedade Brasileira de Educação, 2011. p. 1382-1385.

BAPTISTA, Luciana Ferreira et al. A aplicabilidade de um jogo eltrônico na educação ambiental. In: V WORKSHOP DE PÓS-GRADUAÇÃO E PESQUISA DO CENTRO PAUlA SOUZA, 5., 2010, São Paulo. Anais. São Paulo: Centro Paulo Souza, 2010.

BRUM, Wanderley Pivatto; SILVEIRA, Gisele Aparecida Silva. A utilização do jogo educativo Geonandia 3D no Ensino de Matematica: uma investigação com estudantes de séries iniciais do ensino fundamental sobre o tema geometria. BoEM, v. 2, n. 2, p. 30-55, 2004.

BUCHINGER, Diego; HOUNSELL, Marcelo da Silva; DIAS, Claudinei. Colaboratividade em um Jogo Eletrônico para Ensino sobre Dengue. In: $\mathbf{2 3}^{\mathbf{0}}$ SIMPÓSIO BRASILEIRO DE INFORMÁTICA NA EDUCAÇÃO (SBIE), 23., 2012, Rio de Janeiro. Anais. Rio de Janeiro: UFRJ e UNIRIO, 2012. p. 26-30.

CAMPOS, Jeter S et al. URIHI : Jogo Educativo para Preservação Ambiental na Amazônia. In: CONGRESSO BRASILEIRO DE INFORMÁTICA NA EDUCAÇÃO, 2012, Rio de Janeiro. Anais. Rio de Janeiro: UFRJ e UNIRIO, 2012.

CUNHA, Marcelo Machado et al. Aprendendo sobre vida marinha no contexto de um jogo eletrônico. In: VIII BRAZILIAN SYMPOSIUM ON GAMES AND DIGITAL ENTERTAINMENT, 8., 2009, Rio de Janeiro. Anais. Rio de Janeiro: IEEE Computer Society, 2009. p. 46-55.

DAMBROS, Gabriela et al. A utilização de tecnologias na cartografia escolar: jogo digital para a alfabetização cartográfica. Geosaberes, v. 4, n. 7, p. 4-15, 2013.

DOTTA, Edivani. Elaboração de um Jogo Digital Educacional sobre Saúde Bucal Direcionado para a População Infantil. Pesquisa Brasileira em Odontopediatria e Clínica Integrada, v. 12, n. 2, p. 209-215, 1 jul. 2012.

LEITÃO, Andre Henrique de Brito et al. Terra das Cores: uma proposta de Jogo Educacional Infantil para o exercício do raciocínio lógica-matemático. In: SIMPÓSIO BRASILEIRO DE INFORMÁTICA NA EDUCAÇÃO (SBIE), 23., 2012, Rio de Janeiro. Anais. Rio de Janeiro: UFRJ e UNIRIO, 2012. p. 26-30. 
LOULA, Angelo C et al. Modelagem Ambiental em um Jogo Eletrônico Educativo. In: VIII BRAZILIAN SYMPOSIUM ON GAMES AND DIGITAL ENTERTAINMENT, 2009, Rio de Janeiro. Anais. Rio de Janeiro, 2009. p. 171-180.

LUCENA, Guilherme Leocárdio; AZEVEDO, Meryglaucia Silva. QUIZmica: Um jogo virtual auxiliando o ensino de Química. Revista Tecnologias na Educação, v. 4, n. 7, p. 1-11, 2012.

MACEDO, Daniel V. et al. IntEducaTrânsito: um jogo 3D interativo e educativo sobre as normas de trânsito controlado por dispositivos não tradicionais. In: SBC - SBGAMES, 2013. p. $39-47$.

MELO, Leandro De Almeida; COSTA, Thaíse Kelly De Lima; BATISTA, Any Caroliny Duarte. Pense bem: proposta e desenvolvimento de jogo digital para ensino de computação na educação básica. In: II CONGRESSO BRASILEIRO DE INFORMÁTICA NA EDUCAÇÃ̃O (CBIE 2013), 2., 22 nov. 2013. Campinas. Anais. Campinas: SBC, 2013. p. 346355.

MESQUITA FILHO, Antônio Francisco Gomes de. Soletrandomob: um jogo educacional voltado para o ensino da ortografia na língua portuguesa. In: V CONGRESSO DE PESQUISA E INOVAÇÃO DA REDE NORTE NORDESTE DE EDUCAÇÃO TECNOLÓGICA (CONNEPI 2010), 5., 2010, Maceió. Anais. Maceió: Rede Norte Nordeste de Educação Tecnológica, 2010.

MÜLLER, Raquel M.; CAVALCANTE, Gabriel D.; SANTOS, Leandro A. Laguna Aprendendo sobre a Guerra do Paraguai com jogo educativo. In: XXVII CONGRESSO DA SBC, 17., 2007, Rio de Janeiro. Anais. Rio de Janeiro: Brazilian Computer Society (SBC), 2007. p. 256-263.

SALOMÃO, Samuel Elias da Silva; HEIDRICH, Felipe Etchegaray. Produção de material didático a partir de uma interface de jogo digital. In: GRAPHICA, 2007, Curitiba. Anais. Curitiba: [s.n.], 2007.

SANTANA, Camila et al. Tríade: delineando o processo de construção de um roteiro de um jogo eletrônico Camila Santana. In: VI BRAZILIAN SYMPOSIUM ON COMPUTER GAMES AND DIGITAL ENTERTAINMENT, 6., 2007, São Leopoldo. Anais. São Leopoldo: Brazilian Computer Society, 2007.

SCHETTINO, Raphael Luiz et al. Desenvolvimento do jogo eletrônico educa tivo " Math Fuga" para alunos do ensino fundamental. In: II CONGRESSO INTERNACINAL INTERDISCIPLINAR EM SOCIAIS E HUMANIDADES, 2., 2013, Belo Horizonte. Anais. Belo Horizonte: ANINTER, 2013.

SILVA, Francisco Airton Pereira da. Spacequiz: Um jogo educacional para celular voltado ao ensino de aritm etica. In: VIII BRAZILIAN SYMPOSIUM ON GAMES AND DIGITAL ENTERTAINMENT, 8., 2009, Rio de Janeiro. Anais. Rio de Janeiro: Brazilian Computer Society, 2009. p. 79-82.

SILVA, Ricardo Inacio Alvares; MOTA, Rosilane Ribeiro; BRANCHER, Jacques Duilio. Jogo eletrônico para aprendizado e metaforização de algoritmos recursivos. Semina: Ciências Exatas e Tecnológicas, v. 34, n. 1, p. 45-56, 30 jun. 2013.

SILVA, Sebastian Saullo Ribeiro da; NÓBREGA, Silvia Marques da; JACOB JR, Antonio Fernando Lavareda. Labirinto do Rato : Jogo educacional infantil para dispositivos móveis. In: XXII SBIE - XVII WIE, 2011, Aracajú. Anais. Aracajú: [s.n.], 2011.p. 1407-1410.

SILVA, Valter da et al. Exterminadores de Dengue: Um jogo educativo dinâmico como ferramenta de educação contra a dengue. In: SBGAMES, 2011, Salvador. Anais. Salvador: SBC 2011. p. 7-10.

SOUSA, Jhamerson; GUIMARÃES, Larissa; PIRES, Yomara. "Elimine a dengue": Um jogo educativo interativo que oferece informações para eliminar a dengue. In: NUEVAS IDEAS EN INFORMÁTICA EDUCATIVA, 2012, Santiago. Anais. Santiago: TISE, 2012. p. 403-404.

TRESCASTRO, Lorena Bischoff; SOUZA, Cássia Pinheiro de. Do impresso ao jogo digital: estrategias de leitura da obra O Guarani. Interdisciplinar, v. 21, p. 85-97, 2014. 